\title{
Low Cost Robotic Hand Gloves
}

\author{
Abhijeet S. Taksale ${ }^{1}$, Amol S. Dangale ${ }^{1}$, Imran N. Momin ${ }^{1}$, Sagar V. Sukode ${ }^{2}$ \\ ${ }^{I}$ (Department of Electronic and Tele. Communication Engineering, Symbiosis University, Pune, India) \\ ${ }^{2}$ (Department of Computer Science and Engineering, Symbiosis University, Pune, India)
}

\begin{abstract}
The aim of the paper is to introduce to a low cost robotic hand that can reproduce the abilities and movements of a human hand in order to perform curtain task such as holding, grabbing, etc. Robotic hand was designed to help disabled person, who can able to control the robotic hand with limited physical motion. The robotic hand we designed is under semi autonomous machine. this hand requires commands from the user. We used flex sensors to incur data from human hand, to reproduce the motion in our robotic hand. In this system commands from sensor which senses the actual motion of the human finger and correspondingly sends the signal to the robotic hand. To reach this target we considered three aspects. First, how to develop exo-skeleton of robotic hand in order to manipulate objects like humans do. Second was exploration of proper signals that can be used in order to move the robotic finger. Finally, how to program robotic hand for manipulating different tasks. Details of hand control hardware and software are presented in this paper. Results of the experimental work for different objects are enumerated. Referring this, now we are building a low cost robotic hand gloves which will overcome physical weakness in old people.
\end{abstract}

Keywords: Robotic Hand, Controller, Materials Handling, Flex Sensor.

\section{INTRODUCTION}

Robots have the potential to play a large role in our world. Instead of humans the robots can be assigned with tasks as to avoid the human loss. However, the use for robots grows, so as to interact with objects in the hazardous environment. Human beings interact physically with environment using their hands. Robotic hand systems can be used in robust environments such as those encountered in nuclear, military, chemical and most for space applications.

Number of researchers on the anthropomorphic multi-fingered robotic hand have been reported till date $[1,2,3]$. The MIT hand developed by Jacobsen. is operated by actuators which are located in remote place from the robotic hand frame and attached by tendon cables [4] Jacobsen et al., 1984; Jacobsen et al. 1988). Hirzinger et al. designed DLR-Hand II, which built the actuators into the hand. Each finger of robot hand is attached to a motor, 6-DOF fingertip force torque sensor and integrated electronics along with curtain controller $[5,6]$. Kawasaki et al. developed anthropomorphic robotic hand called the Gifu hand III, which has a thumb and four fingers with the suitable controller [7]. The thumb has 4 joints with 4-DOF and each of the fingers has 4 joints with 3-DOF. Moreover, the assigned tactile sensor which is made of conductive link is arranged about 859 sensing points on the palm and the fingers of the robotic hand. Shimojo et al. used the pressure conductive rubber as a pressure sensing material [8]. They attached the sensor onto fingers of robotic hand and demonstrated its grasping operations with a column, sphere, etc.. Although many researchers have been done already, however, their motion of robotic hands is different from human. The mechanism of robotic hands developed by MIT was different than others because they utilized 32 pneumatic actuators to operate three fingers and a thumb through a bunch of cables [9].

In this paper, we tried to design a robotic hand which is an replica of a human hand. The motions of the human fingers are sense by flex sensor. Here, we have used Atmega 328P which is one of the advance 8 bit controllers in the AVR family. The program and layout or frame appearance on PC done with Codevision Avr_1.25.8 and Proteus 7.6 SP4. In section II of this paper hardware and design is discussed. In the next section we discussed the algorithm used in for this paper. Algorithm is explained well with the help of flow chart. In section IV the implementation of hardware is illustrated. It is then followed with experiment and result what we got after project is discussed. The target was to reproduce human finger motion which are perform in order to hold and grab the object as like human hand. Also force robotic hand as per the human motion.I

\section{HARDWARE DESCRIPTION AND DESIGN}

For the project, a robotic hand made from PVC is used. The signal from the flex sensors are given to a voltage divider circuit and correspondingly signals are sent to the robotic hand. The arrangement is done such that the DC motor can fit in the mechanical assembly properly. To move the fingers of mechanical hand servo motors are used. The motors shafts are fixed with readymade PVC part which will help in holding the finger position. The parts used are shown in Fig 1. This small part will hold the current position until the new digital value is 
received by the motor. Internal assembly of a servo motor is shown in Fig 2. The mechanical finger is connected to the motor with the help of cables. Another important part of this project that was flex sensor. These flex sensors are used in measure the bend in the each Fig. And for the embedded system we used microcontroller.

The microcontroller chosen on basis of required ADCs, PWMs and memory requirements. The selection of controller is also based on the power consumption. To dump the program in microcontroller we used internal flash memory. To program the microcontroller we designed a special programmer which is compatible with the chosen one.

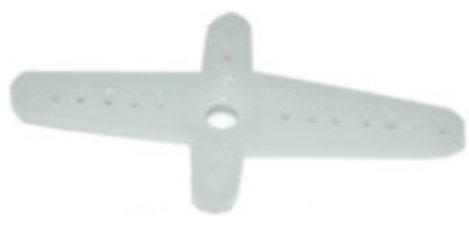

Figure 1: PVC part

The PVC part shown in Fig 1 is mounted on the shaft of the DC servo motor.

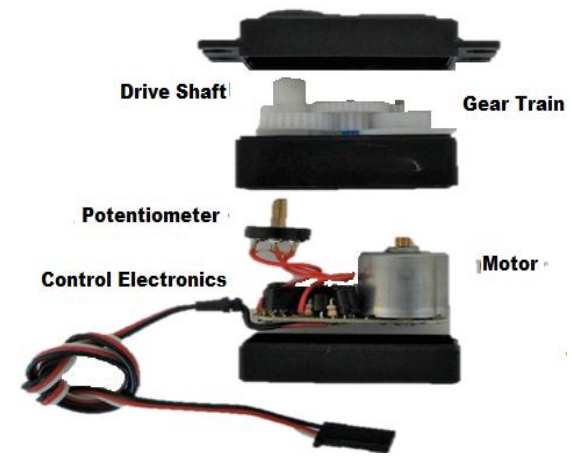

Figure 2: Internal of servo motor

To increase the torque of the dc motor gear assembly is used. It is also used to control speed of dc servo motor. The internal circuit consist of potentiometer register and the special purpose IC's.

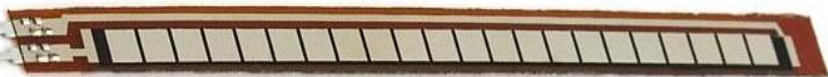

Figure 3: Flex Sensor

Fig 3 shows the flex sensor. It gives the variation in resistance as flex sensor is bent. This variation in resistance is provided to voltage divider circuit. So variation in resistance as converted in voltage variation. This voltage is analog in nature as to convert it into digital ADC present in the controller is used.

\section{IMPLEMENTATION}

An embedded computer controlled five fingered robotic hand which was designed and developed with a simple and minimal control strategy. The approach is based on anthropomorphic design with four fingers and an opposing thumb.

In Fig 4 schematic diagram of robotic hand is shown. The pin 11, 12, 15, 16, 17 of controller are connected to five servo motors. And pin $24,25,26,27,28$ are connected to the flex sensors. Pin 1, 17, 18,19 are used for programming the microcontroller. The power for the robotic hand is provided by the portable battery. The supply we used was a standard $5 \mathrm{~V}, 1$ A battery. 


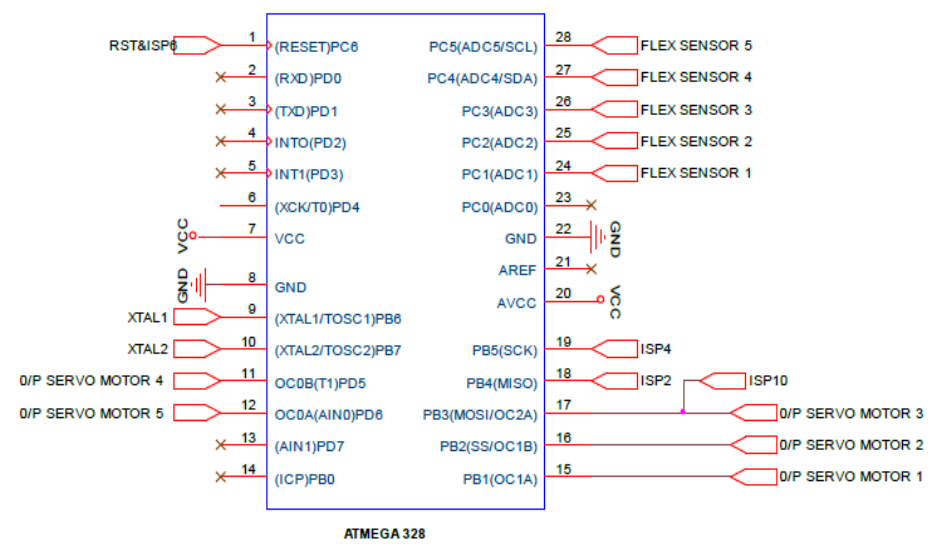

Figure 4: Schematic diagram of the project

While implementing the project we came across the power consumption issue. As there we used a pair of motor and flex sensor for each of the fingers. So we selected such a microcontroller which will fulfill the requirement. Before going on the actual mounting of the component we started testing our algorithm which followed the flow chart Fig 5.

\section{FLOW CHART}

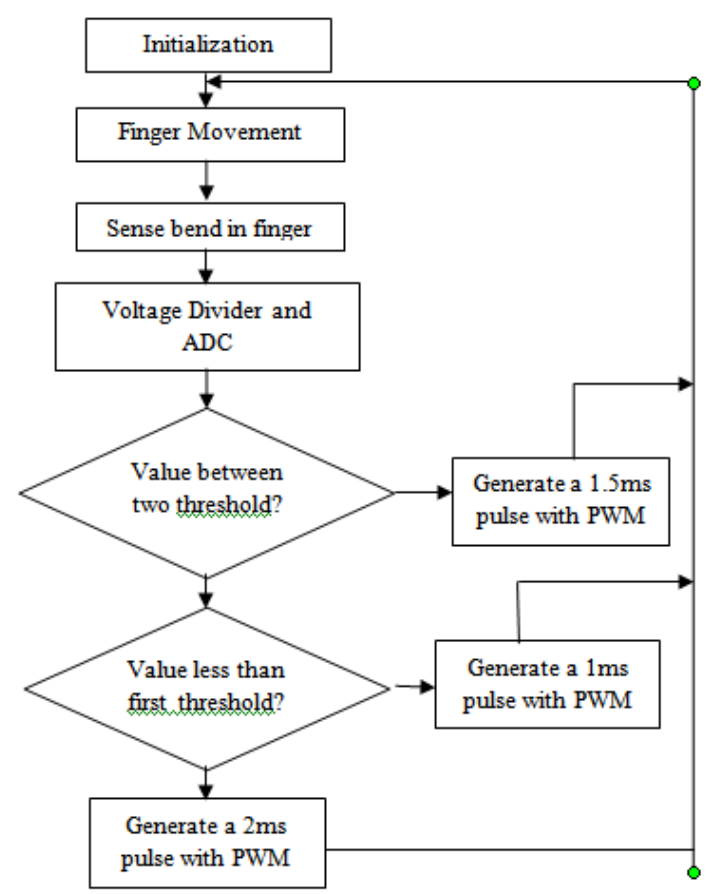

Figure 5: Flow chart for the algorithm

\section{EXPERIMENTAL RESULT}

Before mounting the components each flex sensor is tested with an individual servo motors. As each Fig possess the different threshold value. The digital values from each Fig is observed and displayed in real time using Codevision AVR software. We found the different threshold values for each finger.

So while writing the code in Embedded $\mathrm{C}$ we used the above important observation. The position of each Fig is observed after every 50ms. For this we used a delay program. We program the microcontroller in order to use the five pair of ADC and PWM pin. For dumping the code we use the internal memory of the 8 bit AVR. After testing motors we moved for the PCB design and mounted the components. Once again the board is tested and the experiment is conducted. In Fig 5 the experimental results are shown in the form of photographs. 

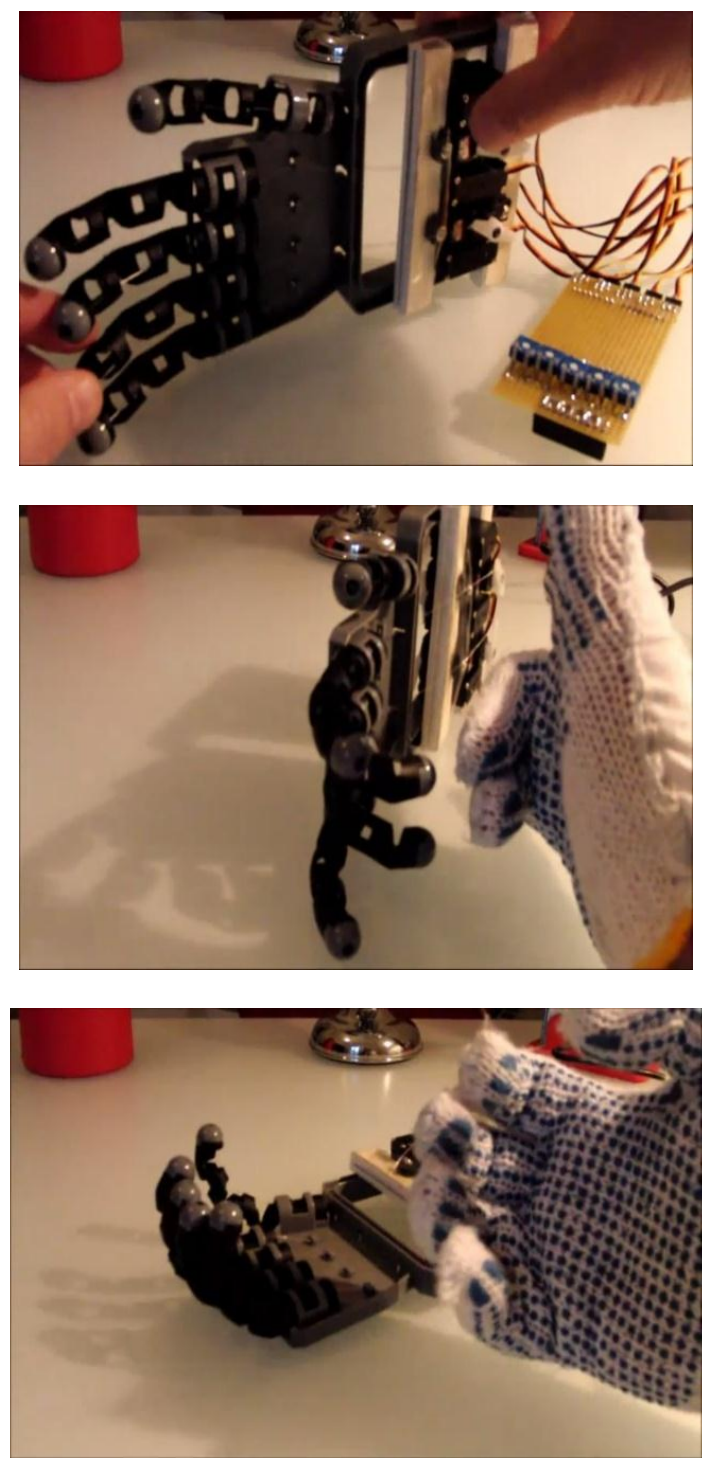

Figure 6: Actual Photograph of the Robotic Hand

\section{DISCUSSION AND FUTURE WORK}

The research on robotic hand is certainly important in development and learning. Our results show how the exploratory procedures, can be used to recognize the position of the finger and accordingly how to command the motors with respect to an object as well as the no-object condition. In the future we will introduce a portable hand gloves which possess the sensor and the motor. Both will be mounted on the gloves along with embedded system. These will increases the power in the grip even less pressure is applied by the figures. This product will be helpful for the old person and person with vitamin D deficiency with leads to weakness.

\section{CONCLUSION}

Robotic hand has been designed, built, and tested completely. The operation of mechanical finger movement in respects of all joints and object detection, grasping and releasing various types of objects are tested. A good repeatability for every task performed is observed. Finger movement is arbitrary and tested the same. To test size restrictions on objects, experiments are performed at grasping spherical objects. Maximum diameter of sphere is $90 \mathrm{~mm}$, and minimum diameter of sphere is $30 \mathrm{~mm}$, is observed for any object. An interesting aspect of this design is that the ranges of weight can be increased by adding more powerful motors and cables of higher tensile strength. This enables the hand to be configured for the application by the selection of the appropriate motors. It has been demonstrated that this hand can grasp a variety of objects with different surface characteristics and shapes without having to reconstruct a surface description of the object. We tried to make exact replica movement of human finger motion. The concept can be used in making a mechanical assembly which can be wear as gloves. This will help the old person to make the proper grip on an object. 


\section{REFERENCES}

[1] Nicholas Thayer and S.Priya. 2011, "Design and implementation of a dexterous anthropomorphic robotic typing (DART) hand", Smart Mater. Struct.20(2011) 035010(12pp), doi:10.1088/0964-1726/20/3/035010.

[2] Cipriani, C., Zaccone, F., Stellin, G., Beccai, L., Cappiello, G., Carrozza, M.C., and Dario, P., 2006, “Closed-loop Controller for a Bio-inspired Multi-fingered Under actuated Prosthesis", Proc. of the 2006 IEEE Int. Conf. on Robotics and Automation, Orlando, FL, USA, May pp. 2111-2116.

[3] Hoshino, K., and Kawabuchi, I., 2006, "Dexterous robot hand with pinching function at fingertips", Proc. of the 1st IEEE/RAS Int. Conf. on Biomedical Robotics and Biomechatronics, BioRob 06, Pisa, Italy, February 20-22.

[4] Jacobsen S C et al 1984 The UTAH/M.I.T. "Dextrous hand: work in progress "Int. J. Robot. Res. pp. 321-50.

[5] M. Fischer, P. Van der Smagt, and G. Hirzinger, "Learning techniques in a dataglove based telemanipulation system for the dlr hand," in Robotics and Automation, Proceedings. 1998 IEEE Int. Conf. on, vol. 2, May 1998, pp. 1603-1608.

[6] H. Hu, J. Li, Z. Xie, B. Wang, B. Liu, and G. Hirzinger, "A robot arm/hand teleoperation system with telepresence and shared control," in Advanced Intellingent Mechatronics, Proceedings, 2005 IEEE/ASME Int. Conf. on, 2005, pp. $1312-1317$.

[7] S. Ekvall and D. Kragic, "Interactive grasp learning based on human demonstration," in Robotics and Automation, Proceedings, 2004 IEEE Int. Conf. on, vol. 4, May 2004, pp. 3519-3524.

[8] J. Aleotti and S. Caselli, "Grasp recognition in virtual reality for robot pre grasp planning by demonstration," in Robotics and Automation, Proceedings, 2006 IEEE Int. Conf. on, 2006, pp. 2801-2806.

[9] M. T. Ciocarlie and P. K. Allen, "Hand posture subspaces for dexterous robotic grasping," The International Journal of Robotics Research, vol. 28, no. 7, pp. 851-867, July 2009.

[10] Yoshikawa, T.: Foundations of Robotics, Analysis and Control, The MIT Press, 1990.

[11] J. Butterfa_, M. Grebenstein, H. Liu and G. Hirzinger," DLR-Hand II: Next Generation of a Dextrous Robot Hand", Proceedings of the IEEE International Conference on Robotics and Automation, Seoul, Korea May 21-26, 2001.

[12] J. Butterfa_, M. Grebenstein, H. Liu and G. Hirzinger," DLR-Hand II: Next Generation of a Dextrous Robot Hand", Proceedings of the IEEE International Conference on Robotics and Automation, Seoul, Korea May 21-26, 2001.

[13] Lovchik, C.S.; Diftler, M.A.: "The Robonaut Hand: A Dexterous Robot Hand for Space", Proceedings of the IEEE Int. Conference on Robotics and Automation, Detroit, 1999, S. 907-912.

[14] A. Miller, P. Allen, V. Santos, F. Valero-Cuevas, "From robotic hands to human hands: a visualization and simulation engine for grasping research", Intelligent Manipulation and Grasping International Conference (IMG'04), Genova, Italy. 1-2 July 2004 